\title{
25 Research Soure \\ Optic Nerve Sheath Diameter in Severe \\ Preeclampsia with Neurologic Features versus Controls
}

Mary E Sterrett ( $\square$ msterret@uw.edu )

University of Washington Medical Center https://orcid.org/0000-0002-1698-4951

\section{Brittany Austin}

Medical University of South Carolina

Ryan Barnes

Medical University of South Carolina

Eugene Chang

Medical University of South Carolina

\section{Research Article}

Keywords: Pregnancy, Preeclampsia, Ultrasonography, Point-of-care systems*, Optic Nerve

Posted Date: February 28th, 2022

DOI: https://doi.org/10.21203/rs.3.rs-1019873/v1

License: (c) (i) This work is licensed under a Creative Commons Attribution 4.0 International License.

Read Full License

Version of Record: A version of this preprint was published at BMC Pregnancy and Childbirth on March 19th, 2022. See the published version at https://doi.org/10.1186/s12884-022-04548-8. 


\section{Abstract}

Background: Optic nerve sheath diameters (ONSD) have been validated as an accurate screening tool to detect elevated intracranial pressure in hypertensive encephalopathy. The neurologic manifestations of preeclampsia and/or eclampsia mimic those of hypertensive encephalopathy. This study was performed to assess the incidence of elevated optic nerve sheath diameters of patients with severe preeclampsia and neurologic criteria compared to non-preeclamptic patients. The secondary objective was to determine baseline optic nerve sheath diameters in patients with severe preeclampsia without neurologic criteria and preeclampsia without severe features.

Methods: Single site cohort study including 62 pregnant women 18 years or older and 20 weeks or further gestation. Patients with preeclampsia without severe features, preeclampsia with severe features by nonneurologic criteria, preeclampsia with severe features with neurologic criteria, and patients without preeclampsia were enrolled via convenience sampling. One blinded reviewer measured sheath diameters; baseline demographics and pregnancy data were collected by chart review. Statistical analysis was completed with STATA/IC 16. Categorical variables were compared by the $\chi^{2}$ test. Continuous variables were presented as mean \pm standard deviation, and discrete variables were presented as medians and compared by Kruskal-Wallis testing. Normality was confirmed by Shapiro-Wilk testing. Linear and logistic regression were used to test the association between the preeclampsia groups and optic nerve sheath diameters. Models were presented as unadjusted and adjusted for BMl, gestation, hypertension, diabetes, parity, and gravidity.

Results: The incidence of optic nerve sheath diameters $>5.8 \mathrm{~mm}$ was $43.8 \%$ in the severe preeclampsia with neurologic features cohort, and $42.1 \%$ in the control cohort, with a relative risk of 1.04 . Patients with severe preeclampsia without neurologic features had sheath diameters of $5.75 \mathrm{~mm}+/-1.09 \mathrm{~mm}$; nonsevere preeclampsia patients had sheath diameters of $5.54 \mathrm{~mm}+/-1.26 \mathrm{~mm}$.

Conclusions:

We did not find a significant elevated optic nerve sheath diameter relative risk between severe preeclampsia patients with neurologic features and non-preeclampsia control patients. This is the first study to assess a North American population utilizing ACOG criteria for severe and non-severe preeclampsia, with severe cohorts additionally stratified by neurologic criteria.

\section{Background}

Preeclampsia (PE) is an obstetrical complication characterized by new-onset hypertension or superimposed hypertension with or without one systemic condition including proteinuria, hepatic dysfunction, neurologic symptoms, renal insufficiency, pulmonary edema, or thrombocytopenia during the second half of pregnancy. 
The clinical manifestations of severe preeclampsia can be confusing for the clinician, as several subjective symptoms such as headache, epigastric pain, or right upper quadrant pain can be benign findings in pregnancy. Although hypertension in pregnancy is acknowledged to be a continuum of risk, and early delivery is indicated for many women, identifying an objective measure of lower risk could be useful in stratifying the need for early term delivery to avoid complications of premature delivery, and subsequent maternal management.

The clinical neurologic manifestations of preeclampsia and/or eclampsia have multiple theories about pathogenesis. One theory is that hyper perfusion of the brain due to decreased cerebrovascular resistance leads to vasogenic edema, similar to hypertensive encephalopathy $(1,2)$. Hypertensive encephalopathy is hypothesized to be an overperfusion injury causing disordered cerebral autoregulation and subsequent globally decreased cerebral blood flow with extravasation of fluid into the cerebral parenchyma(3,4). Similarly, the results of noninvasive studies of cerebral blood flow and resistance suggest that vascular barotrauma and loss of cerebral vascular autoregulation contribute to the cerebral vascular pathology in preeclampsia(5-7). Radiologically, this can be visualized via computed tomography and magnetic resonance imaging demonstrating cerebral edema in some women with severe preeclampsia or eclampsia(1).

Cerebral edema is believed to cause this increase in intracranial pressure. The subarachnoid spaces surrounding the optic nerve communicate with the intracranial cavity, and changes in cerebrospinal fluid pressure are transmitted along the distensible optic nerve sheath to increase sheath diameter(8, 9). Two recent meta-analyses suggest that optic nerve sheath diameters can be used as surrogate markers for elevated intracranial pressure, but cite a range of cut-off from 4.8 to $6.3 \mathrm{~mm}$ based on the multiple studies which were included $(10,11)$. The wide range in potentially significant ONSD measurements raises the question of what an expected ONSD in an obstetric population is. In a comparison of ocular ultrasonography with gold standard measures of intracranial pressure (ICP) via invasive devices such as intraventricular catheters, values of ONSD above $5.8 \mathrm{~mm}$ were shown to be associated with a $95 \%$ risk of raised ICP (i.e. more than $20 \mathrm{mmHg}$ )(12), which is why we chose to use this threshold, but the optimal ONSD cuff off value for raised ICP is unknown.

To date, there have been four studies evaluating ONSD in preeclampsia and eclampsia. Dubost et al evaluated a total of 51 women: 26 preeclamptic patients, of which 13 had severe preeclampsia, and 13 had preeclampsia without severe features. A self-reported weakness of the Dubost study was the small percentage of severe preeclampsia patients with neurologic symptoms, limiting the ability to determine a relationship with ONSD enlargement(13). Only 8 women with preeclampsia had headaches, which included both severe $(n=7)$ and non-severe preeclamptic $(n=1)$ patients. The sample included preeclampsia patients predominantly diagnosed (54\%) with severe features by renal dysfunction, which is reported only in approximately $1 \%$ of women with severe preeclampsia(14). They found that approximately $19 \%$ of severe preeclamptic patients had ONSD values indicating intracranial pressures above $20 \mathrm{mmHg}(13)$. 
Simenc et al assessed 30 severe preeclampsia patients and compared their ONSD and optic disc height (ODH) measurements to control patients(15). They did not assess preeclamptics without severe features or differentiate patients by subjective or objective features of severe preeclampsia. They found $43 \%$ of patients with severe preeclampsia had ONSD measurements $>5.8 \mathrm{~mm}$ and $77 \%$ with an ODH $\geq 1 \mathrm{~mm}$, compatible with intracranial hypertension(15). Ortner et al evaluated point of care ultrasound (POCUS) in 95 severe preeclampsia patients, which included the incidence of elevated ONSD in this population(16). However, their primary outcome was the relationship of albumin to POCUS for pulmonary edema or elevated ONSD above $5.8 \mathrm{~mm}(16)$. Singh and Bhatia evaluated 75 pregnant patients, in cohorts of 25 patients of severe preeclampsia, eclampsia, and control groups. They found significant differences between the ONSD measurements in the three cohorts with $44 \%$ of preeclampsia patients and $66 \%$ of eclampsia patients demonstrating elevations of ONSD values $\geq 5.7 \mathrm{~mm}(17)$. Of note, they had included patients with IUGR for diagnosis of severe preeclampsia, and excluded those with blurred vision, moderate-to-severe renal or hepatic dysfunction or coagulopathy(17). Exclusion of patients with laboratory criteria for severe preeclampsia would remove $7.3 \%$ of patients with preeclampsia, especially as the frequency of abnormal laboratory values in women with pregnancy-associated hypertension increases with disease severity(18). Evaluating ONSDs in a North American population with severe preeclampsia by ACOG criteria is a necessary step to begin assessing utility of this point of care technique to our obstetric population.

This aim of this study was to estimate the incidence of elevated ONSD in severe preeclampsia patients with neurologic features compared to non preeclamptic patients. The secondary objective was to determine baseline optic nerve sheath diameters in patients with severe preeclampsia with and without neurologic criteria and preeclampsia without severe features. We anticipated correlation of clinical features of severe preeclampsia to elevated ONSD representing vasogenic edema.

\section{Methods}

\section{Design}

This was a prospective study including four pregnant cohorts: patients without preeclampsia ("controls"); patients with PE without severe features; patients with severe PE (SPE) but no neurologic findings, and patients with SPE and neurologic findings. Recruitment began in November 2018 and continued until January 2020, when access to the study-specific ultrasound was expected to cease. Participants were recruited from the inpatient or antepartum unit of Labor and Delivery at a major university hospital in a southern state.

\section{Sample}

Patients were enrolled by convenience sampling. Inclusion criteria included women with a singleton, nonanomalous pregnancy, participants $>20$ weeks gestation, and 18 years old or older at the time of recruitment. Exclusion criteria included patients with preexisting eye conditions or ocular surgeries as well 
as any woman with pseudotumor, intracranial hypertension, seizure disorder, or known intracranial pathology.

Preeclampsia by ACOG criteria is traditionally defined by a patient having two elevated blood pressures of $140 / 90$ four hours apart with a urine protein to creatinine ratio of 0.3 or higher. If the patient did not meet urinary protein criteria, they could also be diagnosed by severe features, including elevated liver enzymes to twice the upper limit of normal, elevated creatinine of greater than $1.1 \mathrm{mg} / \mathrm{dL}$, pulmonary edema, newonset headache unresponsive to acetaminophen, visual disturbances, severe persistent right upper quadrant or epigastric pain, a platelet count less than $100 \times 109 / \mathrm{L}$, or severe range blood pressures of $160 \mathrm{~mm} \mathrm{Hg}$ or higher, diastolic blood pressure of $110 \mathrm{~mm} \mathrm{Hg}$ or higher.

We powered this study by assuming a relative risk of 8.6(15), expected incidence of elevated ONSD in non-preeclamptic patients of $5 \%(19)$, confidence level of 0.95 , and a power of 0.8 . This required 16 patients in each cohort.

\section{Data Collection}

Patients were interviewed at the time of enrollment and asked their age, ethnicity, and history of intracranial pathology. BMI, blood pressures, gravidity and parity, and gestational age were recorded from their medical record. Subjects were asked about symptoms of severe preeclampsia, including persistent headaches, visual scotomata or changes, right upper quadrant or epigastric pain, and shortness of breath that would indicate pulmonary edema. Informed consent was obtained in writing with an IRB-approved form.

Subjects were placed in a semi-recumbent position with the upper part of the body and head at 30-45 degrees from the vertical position for three minutes or less to obtain ultrasound data. A thick layer of water-soluble gel was applied to the orbital fossa with the eye closed, in accordance with prior published protocols(20-22). A single ultrasonographer (M.S.) previously trained in obtaining optic nerve sheath images by R.B. performed all ultrasound scans. A 13-6 MHz linear probe (L25/13-6 MHz, of Fujifilm SonoSite, Bothell, WA) was utilized $(23,24)$. The transducer was gently placed over the fossa to visualize the globe and surrounding structures(25). Multiple still images of each eye were saved for subsequent review and measurement.

An attending emergency room physician (R.B.) trained in ocular ultrasound performed a blinded assessment of de-identified images to obtain ONSD measurements. This was done in a post hoc fashion directly on the ultrasound machine. ONSD was measured $3 \mathrm{~mm}$ behind the globe using an electronic caliper at an axis perpendicular to the optic nerve. Two ONSD measurements were taken in each eye, and the mean of the measurements was used. If the two measurements could not be taken, then one data point was used instead of a mean. A maximum of four values were taken, and the mean values for each eye, or one value if only one clear image was obtained, was stored for data analysis.

\section{Statistical Analysis}


Statistical analysis was completed with STATA/IC 16 (StataCorp. 2019. Stata Statistical Software: Release 16. College Station, TX: StataCorp LLC). We powered our study using the elevated ONSD incidence of $43 \%$ from Simenc et al (15), and an anticipated baseline elevated ONSD rate of 5\%(19). To obtain a power of $95 \%$, using an alpha level of 0.05 , we required 16 patients in our severe preeclampsia cohort with neurologic features and 16 in our non-preeclamptic cohort to assess the primary outcome of significant difference in ONSD means between the two groups. Our secondary outcome was to assess if there was a significant difference between any of the cohorts' ONSD means.

Categorical variables of chronic hypertension (cHTN) and preexisting diabetes (DM) were presented as total numbers and compared by the $\chi^{2}$ test. Continuous variables of gestational age and BMI were presented as mean \pm standard deviation, and discrete variables of gravidity and parity were presented as medians and compared by Kruskal-Wallis testing. The normality of the ONSD data was confirmed by Shapiro-Wilk testing.

Linear and logistic regression was used to test the association between the preeclampsia groups and ONSD. Models were presented as unadjusted and adjusted for BMI, gestation, hypertension, diabetes, parity, and gravidity. The incidence of elevated ONSD in each group was calculated, and the relative risk of elevated ONSD was assessed. Statistical analysis was completed with STATA/IC 16 (StataCorp. 2019. Stata Statistical Software: Release 16. College Station, TX: StataCorp LLC.

\section{Results}

A total of 62 patients were enrolled. Demographic data is included in Table l: Patient Demographics. We adjusted for BMI, gestation, cHTN, DM, parity, \& gravidity and found no significant difference during our regression analysis. 
Table 1

Patient Demographics

\begin{tabular}{|lllllll|}
\hline & $\begin{array}{l}\text { Total } \\
\mathbf{n}=62\end{array}$ & $\begin{array}{l}\text { sPE with } \\
\text { Neurologic } \\
\text { Features } \\
\mathbf{n}=16\end{array}$ & $\begin{array}{l}\text { sPE without } \\
\text { Neurologic } \\
\text { Features } \\
\mathbf{n}=19\end{array}$ & $\begin{array}{l}\text { Preeclampsia } \\
\mathbf{n}=8\end{array}$ & $\begin{array}{l}\text { Controls } \\
\mathbf{n}=19\end{array}$ & $\mathrm{p}$-value \\
\hline $\begin{array}{l}\mathrm{BMI} \\
\left(\mathrm{kg} / \mathrm{m}^{2}\right)\end{array}$ & $\begin{array}{l}34.24 \\
\pm 8.9\end{array}$ & $32.40 \pm 8.01$ & $36.59 \pm 9.24$ & $34.54 \pm 11.20$ & $33.26 \pm 8.90$ & $\mathrm{p}=0.53^{\ddagger}$ \\
\hline $\begin{array}{l}\text { Gestation } \\
\text { (weeks) }\end{array}$ & 34.25 & $31.4 \pm 3.11$ & $32.57 \pm 4.50$ & $35.25 \pm 3.66$ & $37.8 \pm 3.91$ & $\mathrm{p}=0.12^{\ddagger}$ \\
\hline $\begin{array}{l}\text { Chronic } \\
\text { HTN }\end{array}$ & 11 & $4(25 \%)$ & $5(26.3 \%)$ & 0 & $2(10.5 \%)$ & $\mathrm{p}=0.27^{\dagger}$ \\
\hline $\begin{array}{l}\text { Preexisting } \\
\text { DM }\end{array}$ & 9 & $1(6.3 \%)$ & $4(21.1 \%)$ & $1(12.5 \%)$ & $3(15.8 \%)$ & $\mathrm{p}=0.44^{\dagger}$ \\
\hline Gravidity & 2 & 1.5 & 2 & 1 & 2 & $\mathrm{p}=0.30^{*}$ \\
\hline Parity & 0 & 0 & 1 & 0 & 0.5 & $\mathrm{p}=0.27^{*}$ \\
\hline
\end{tabular}

Legend: sPE: Severe preeclampsia. HTN: hypertension. DM: diabetes mellitus

${ }^{\dagger}$ Compared by $x 2$

${ }^{\ddagger}$ Compared by one way ANOVA

${ }^{*}$ Compared by Kruskal-Wallis

For the purposes of our study, an elevated ONSD was defined as a measurement $>5.8 \mathrm{~mm}$. The mean ONSD with confidence intervals and incidence of elevated ONSD was calculated for each cohort, and unadjusted and adjusted results are shown in Table 2. The incidence of elevated ONSD in the severe preeclampsia cohort with neurologic features was $44 \%$, and the incidence in the non-preeclampsia control group was $44 \%$. The relative risk for having an elevated ONSD with severe preeclampsia with neurologic features was 1.04. We also calculated the odds ratio, which resulted in an OR of 0.97 for having an elevated ONSD for the severe preeclampsia cohort with neurologic features. For example, we would expect, on average, that those in the SPE with neurologic features cohort to have an ONSD of $0.26 \mathrm{~mm}$ lower than the patients without preeclampsia (control) cohort. (Table 2) 
Table 2

Association of preeclampsia cohorts with ONSD

Outcome Continuous ONSD

$=$

\begin{tabular}{|c|c|c|c|c|c|c|}
\hline & n & Mean & Unadjusted & & Adjusted * & \\
\hline & & & $\beta(95 \% \mathrm{Cl})$ & p-value & p-value & $\begin{array}{l}\mathrm{p}- \\
\text { value }\end{array}$ \\
\hline sPE with Neuro & 16 & $\begin{array}{l}5.67 \\
(0.78)\end{array}$ & $-0.26(-0.92,0.40)$ & 0.440 & $\begin{array}{l}-0.37(-1.19 \\
0.45)\end{array}$ & 0.367 \\
\hline $\begin{array}{l}\text { SPE without } \\
\text { Neuro }\end{array}$ & 19 & $\begin{array}{l}5.75 \\
(1.08)\end{array}$ & $-0.17(-0.80,0.46)$ & 0.593 & $\begin{array}{l}-0.25(-1.01 \\
0.51)\end{array}$ & 0.506 \\
\hline Mild PE & 9 & $\begin{array}{l}5.51 \\
(1.18)\end{array}$ & '-0.41 (-1.20, 0.37) & 0.299 & $\begin{array}{l}-0.58(-1.43, \\
0.28)\end{array}$ & 0.184 \\
\hline \multirow[t]{4}{*}{ Normal } & 18 & $\begin{array}{l}5.92 \\
(0.84)\end{array}$ & 0 (ref) & & 0 (ref) & \\
\hline & & $\begin{array}{l}\text { Outcome } \\
=\end{array}$ & $\begin{array}{l}\text { Dichotomous } \\
\text { ONSD }\end{array}$ & $>5.8 \mathrm{~mm}$ & & \\
\hline & n & $\begin{array}{l}n>5.80 \\
(\%)\end{array}$ & Unadjusted & & Adjusted * & \\
\hline & & & OR $(95 \% \mathrm{Cl})$ & p-value & OR $(95 \% \mathrm{Cl})$ & $\begin{array}{l}\text { p- } \\
\text { value }\end{array}$ \\
\hline sPE with Neuro & 16 & $7(44 \%)$ & $0.97(0.25,3.78)$ & 0.968 & $0.79(0.14,4.31)$ & 0.783 \\
\hline $\begin{array}{l}\text { SPE without } \\
\text { Neuro }\end{array}$ & 19 & $10(53 \%)$ & $1.39(0.38,5.07)$ & 0.619 & $1.24(0.27,5.80)$ & 0.785 \\
\hline Mild PE & 9 & $1(11 \%)$ & $0.16(0.02,1.52)$ & 0.110 & $0.13(0.01,1.35)$ & 0.087 \\
\hline Normal & 18 & $8(44 \%)$ & 1.00 (ref) & & 1.00 (ref) & \\
\hline \multicolumn{7}{|c|}{ *adjusted for BMI, gestation, cHTN, DM, parity, \& gravidity } \\
\hline \multicolumn{7}{|c|}{$\begin{array}{l}\text { There was not a statistically significant difference in ONSD between the four groups, } \chi 2(3)=3.56, p= \\
0.313 \text {. The control group had the largest diameters without significant difference in ONSD means } \\
\text { between cohorts. Severe preeclampsia with neurologic features had overlap in ONSD measurements } \\
\text { with all three cohorts. (Graph 1) }\end{array}$} \\
\hline
\end{tabular}

\section{Discussion}

The ONSD has been validated as an accurate screening tool to detect elevated intracranial pressure in patients with idiopathic intracranial hypertension, traumatic brain injury, and spontaneous intracranial hemorrhage $(26,27)$. 
This study's lack of a significant increase in ONSD measurements in the severe preeclampsia cohorts was an unexpected outcome, as Simenc et al's study noted a significant increase in the percentage of ONSD measurements $>5.8 \mathrm{~mm}$ in severe preeclampsia groups compared to controls(15). Limitations of the size of the cohorts could be causing a type II error regarding this outcome.

Belfort et al have studied the use of transcranial Doppler and shown that a majority of women with preeclampsia have altered cerebral perfusion pressure (CPP) (28) although the etiology may be different between mild and severe disease states. Specifically, women with mild preeclampsia had reduced CPP compared to normal pregnant women, compared to an increase in CPP for women with severe preeclampsia(28). The authors' theory was that in preeclampsia without severe features, the predominant abnormality was hypoperfusion (suggestive of vasospasm and ischemia)(2), and in severe preeclampsia women had hyper perfusion (suggestive of hypertensive encephalopathy) $(2,28)$. Their findings track with our results of women with preeclampsia without severe features having the overall lowest mean ONSD of $5.54 \mathrm{~mm}$, although the differences in ONSD measurements between our cohorts was not significant. The preeclampsia without severe features cohort had only one patient with an ONSD of $>5.8 \mathrm{~mm}$, giving an incidence of $12.5 \%$ for an elevated ONSD measurement consistent with elevated ICP.

\section{Limitations and Strengths}

This is the first study to assess a North American population utilizing ACOG criteria for non-severe and severe preeclampsia stratified by neurologic criteria. Prior studies used inclusion criteria (such as inclusion by fetal growth restriction) that is not endorsed by ACOG for defining severe versus non-severe preeclampsia(17), varying criteria for defining elevated ONSD in preeclampsia cohorts(15), or lacked cohorts to assess differences by neurologic criteria(13).

As preeclampsia has similar imaging findings as hypertensive encephalopathy, utilizing ONSD as a marker of severity for preeclampsia as for hypertensive encephalopathy is biologically plausible. The physiology of raised intracranial pressure with vasogenic edema from preeclampsia would logically apply to the use of ocular ultrasound as an objective measure of severity. All images were obtained by a single sonographer, removing inter-observer variability.

The limitations of this research include being a single site study. Additionally, a cut-off value for ONSD, such as above $5.8 \mathrm{~mm}$, has not been validated in an obstetric cohort to predict increased ICP. Assessing ONSD prior to the initiation of antihypertensive medications and magnesium or assessing pre- and postmagnesium values would control for a common intervention which could have impacted measurements. It has been found in prior studies on non-pregnant adults that there are rapid changes in ONSD with changing cerebrospinal fluid pressure(29). However, prior studies have found that CPP(30) can be pathologically elevated even after treatment of elevated blood pressure for several days(15), which would make future endeavors consider timing of treatment as a variable, but not a contraindication to obtaining an ONSD measurement. Additionally, there is a lack of comparison to direct ICP measurements, or indirect measurements with magnetic resonance imaging(26). 
Unfortunately, as we enrolled by convenience sampling, self-selection bias is a possible confounder, and chronic hypertension was not controlled for during enrollment. It is known that the background rate of intracranial hypertension is higher in women with chronic hypertension and obesity(31). Although BMI was not found to be significantly different between groups, the incidence of chronic hypertension was variable between the cohorts.

The use of the linear probe may not be available in all labor and delivery units, and evaluation with use of the more common curvilinear probe would be of interest. However, studies comparing measurements made in the visual axis versus the coronal axis have shown significant differences when using a curvilinear probe(32) and decreased variability in ONSD with different axis of measurement found when using a linear transducer(33). There are limited studies assessing standard error of optic nerve sheath ultrasound images, as the acoustic shadow for any optic nerve sheath would be expected to have a consistent error for any measured ONSD with the same probe. However, the error has been assumed to be less than 0.1 to $0.2 \mathrm{~mm}$ by ONSD models utilizing Sonosite L25 linear probes(34).

Obtaining larger sample sizes would improve the ability to control for potential confounders, such as timing of antihypertensive medications or use of magnesium, chronic hypertension(31), class III obesity, age(8), or to assess for racial variations, which have been found to be risk factors for the development of preeclampsia itself $(35,36)$. A powered sample size for each group would also improve the sensitivity of finding a difference in ONSD magnitude between severe preeclampsia, preeclampsia without severe features, and control cohorts, if there is indeed one between the groups. Finally, obtaining baseline measurements of ONSD in pregnant women in all three trimesters would allow for improved interpretation of ONSD in future studies.

\section{Conclusion}

Optic nerve sheath diameters were not found to be significantly different between severe preeclampsia patients with neurologic features and healthy control patients, nor was there a significant difference between any of the cohorts' ONSD means.

This study does not support that ONSD is associated with hypertensive disease severity. Continuing to search for an objective measure to differentiate severe preeclampsia from a chronic hypertension exacerbation would be clinically advantageous, as several subjective symptoms such as headache, visual changes, or right upper quadrant pain can be benign findings in pregnancy. Hypertension in pregnancy is acknowledged to carry a spectrum of risk, and early delivery is indicated for many women. However, identifying an objective measure of risk could be useful in stratifying the need for early term delivery to avoid complications of premature delivery and for maternal management.

\section{Abbreviations}

ONSD 
Optic Nerve Sheath Diameter

ACOG

American College of Obstetrics and Gynecology

PE

Preeclampsia

SPE

Severe Preeclampsia

MUSC

Medical University of South Carolina

PRES

Posterior reversible encephalopathy syndrome

HTN

hypertension

DM

diabetes mellitus

\section{Declarations}

Ethics approval and consent to participate

Medical University of South Carolina Institutional Review Board for Human Research (IRB) Ethics Approval: 11/7/2018 for Project Reference: Pro00078977

All participants gave written consent with an MUSC IRB approved consent form

Consent for publication

All authors consent to publication of the manuscript as written above

Availability of data and materials

Datasets used and analyzed for the current study are available from the corresponding author on reasonable request, and all data analyzed during this study are included in this article

Competing interests

The authors report no conflict of interest and no competing interests.

Funding

No financial support or funding sources.

Authors' contributions 
MS made substantial contributions to conception, the acquisition of data, analysis, and writing of the manuscript. BA made substantial contributions to the acquisition and writing of the manuscript. RB made substantial contributions to the acquisition, interpretation, and writing of the manuscript. EC made substantial contributions to the conception of the project and writing of the manuscript.

Acknowledgements

No additional acknowledgements

\section{References}

1. Schwartz RB, Feske SK, Polak JF, DeGirolami U, laia A, Beckner KM, et al. Preeclampsia-eclampsia: clinical and neuroradiographic correlates and insights into the pathogenesis of hypertensive encephalopathy. Radiology. 2000;217(2):371-6.

2. Hammer ES, Cipolla MJ. Cerebrovascular Dysfunction in Preeclamptic Pregnancies. Curr Hypertens Rep. 2015;17(8):64.

3. Aminoff MJ, Josephson SA. Aminoff's neurology and general medicine. 5th ed.: Amsterdam. Elsevier.; 2014.

4. Bar B. Hypertensive Encephalopathy. Primer on Cerebrovascular Diseases. 2nd ed.: Elsevier.; 2017. pp. 733-7.

5. Belfort M, Saade G, Grunewald C, Dildy G, Abedejos P, Herd J, et al. Association of cerebral perfusion pressure with headache in women with pre-eclampsia. Br J Obstet Gynaecol. 1999;106(8):814-21.

6. Belfort M, Tooke-Miller C, Allen JJ, Varner M, Grunewald C, Nisell H, et al. Pregnant women with chronic hypertension and superimposed pre-eclampsia have high cerebral perfusion pressure. BJOG. 2001;108(11):1141-7.

7. Belfort $M$, Varner M, Dizon-Townson D, Grunewald $C$, Nisell $H$. Cerebral perfusion pressure, and not cerebral blood flow, may be the critical determinant of intracranial injury in preeclampsia: a new hypothesis. Am J Obstet Gynecol. 2002;187(3):626-34.

8. Kristiansson H, Nissborg E, Bartek J Jr, Andresen M, Reinstrup P, Romner B. Measuring elevated intracranial pressure through noninvasive methods: a review of the literature. $J$ Neurosurg Anesthesiol. 2013;25(4):372-85.

9. Blaivas M, Theodoro D, Sierzenski P. Elevated intracranial pressure detected by bedside emergency ultrasonography of the optic nerve sheath. Acad Emerg Med. 2003;10:376-81.

10. E JD, T G J. M M, B K. Ultrasonography of optic nerve sheath diameter for detection of raised intracranial pressure: A systematic review and meta-analysis. Intensive Care Med. 2011;37:1059-68.

11. Robba C, Santori G, Czosnyka M, Corradi F, Bragazzi N, Padayachy L, et al. Optic nerve sheath diameter measured sonographically as non-invasive estimator of intracranial pressure: a systematic review and meta-analysis. Intensive Care Med. 2018;44:1284-94. 
12. Geeraerts T, Merceron S, Benhamou D, Vigue B, Duranteau J. Non-invasive assessment of intracranial pressure using ocular sonography in neurocritical care patients. Intensive Care Med. 2008;34:206267.

13. Dubost C, Le Gouez A, Jouffroy V, Roger-Christoph S, Benhamou D, Mercier F, et al. Optic Nerve Sheath Diameter Used as Ultrasonographic Assessment of the Incidence of Raised Intracranial Pressure in Preeclampsia. A Pilot Study. Anesthesiology. 2012.

14. Acharya A, Santos J, Linde B, Anis K. Acute Kidney Injury in Pregnancy-Current Status. Advances in Chronic Kidney Disease. 2013;20(3).

15. Brzan Simenc G, Ambrozic J, Prokselj K, Tul N, Cvijic M, Mirkovic T, et al. Ocular ultrasonography for diagnosing increased intracranial pressure in patients with severe preeclampsia. Int J Obstet Anesth. 2018;36:49-55.

16. Ortner CM, Krishnamoorthy V, Neethling E, Flint M, Swanevelder JL, Lombard C, et al. Point-of-Care Ultrasound Abnormalities in Late-Onset Severe Preeclampsia: Prevalence and Association With Serum Albumin and Brain Natriuretic Peptide. Anesth Analg. 128. United States2019. p. 1208-16.

17. Singh SK, Bhatia K. Ultrasonographic Optic Nerve Sheath Diameter as a Surrogate Measure of Raised Intracranial Pressure in Severe Pregnancy-induced Hypertension Patients. Anesth Essays Res. 2018;12(1):42-6.

18. Cantu J, Clifton RG, Roberts JM, Leveno KJ, Myatt L, Reddy UM, et al. Laboratory abnormalities in pregnancy-associated hypertension: frequency and association with pregnancy outcomes. Obstet Gynecol. 2014;124(5):933-40.

19. Chen H, Ding G-S, Zhao Y-C, Yu R-G, Zhou J-X. Ultrasound measurement of optic nerve diameter and optic nerve sheath diameter in healthy Chinese adults. BMC Neurology. 2015;15.

20. Helmke K, Hansen HC. Fundamentals of transorbital sonographic evaluation of optic nerve sheath expansion under intracranial hypertension. I. Experimental study. Pediatr Radiol. 1996;26(10):701-5.

21. Helmke K, Hansen HC. Fundamentals of transorbital sonographic evaluation of optic nerve sheath expansion under intracranial hypertension II. Patient study. Pediatr Radiol. 1996;26(10):706-10.

22. Blaivas $M$, Theodoro D, Sierzenski PR. A study of bedside ocular ultrasonography in the emergency department. Acad Emerg Med. 2002;9(8):791-9.

23. FDA. 510(k) K133454.. In: FDA, editor. Online2016.

24. K142017. [Internet]. FDA. 2014. Available from: https://www.accessdata.fda.gov/cdrh_docs/pdf14/K142017.pdf.

25. Geeraerts T, Merceron S, Benhamou D, Vigué B, Duranteau J. Non-invasive assessment of intracranial pressure using ocular sonography in neurocritical care patients. Intensive Care Med. 2008;34(11):2062-7.

26. Ortner CM, Macias P, Neethling E, Krishnamoorthy V, Carvalho B, Swanevelder JL, et al. Ocular sonography in pre-eclampsia: a simple technique to detect raised intracranial pressure? Int J Obstet Anesth. 2020;41:1-6. 
27. Wang J, Li K, Li H, Ji C, Wu Z, Chen H, et al. Ultrasonographic optic nerve sheath diameter correlation with ICP and accuracy as a tool for noninvasive surrogate ICP measurement in patients with decompressive craniotomy. Journal of Neurosurgery. 2019.

28. Belfort MA, Grunewald C, Saade GR, Varner M, Nisell H. Preeclampsia may cause both overperfusion and underperfusion of the brain: a cerebral perfusion based model. Acta Obstet Gynecol Scand. 1999;78(7):586-91.

29. Hans-Christian $\mathrm{H}$, Knut $\mathrm{H}$. Validation of the optic nerve sheath response to changing cerebrospinal fluid pressure: ultrasound findings during intrathecal infusion tests. J Neurosurg. 1997;87(1):34-40.

30. Sonneveld M, Brussé I, Duvekot J, Steegers E, Grune F, Visser G. Cerebral perfusion pressure in women with preeclampsia is elevated even after treatment of elevated blood pressure. Acta Obstet Gynecol Scand. 2014;93(5).

31. MF. H SS, A. K. Intracranial Hypertension. Internet: Treasure Island, FL; 2021 [StatPearls]. Available from: https://www.ncbi.nlm.nih.gov/books/NBK507811/.

32. Blehar DJ, Gaspari RJ, Montoya A, Calderon R. Correlation of visual axis and coronal axis measurements of the optic nerve sheath diameter. J Ultrasound Med. 2008;27(3):407-11.

33. Amini R, Stolz LA, Patanwala AE, Adhikari S. Coronal Axis Measurement of the Optic Nerve Sheath Diameter Using a Linear Transducer. J Ultrasound Med. 2015;34(9):1607-12.

34. Zeiler FA, Unger B, Kramer AH, Kirkpatrick AW, Gillman LA. A Unique Model for Ultrasound Assessment of Optic Nerve Sheath Diameter. Can J Neurol Sci. 2013;40:225-9.

35. Task Force on Hypertension in Pregnancy. Hypertension in Pregnancy. ACOG.org: American College of Obstetrics and Gynecology; 2013.

36. ACOG. ACOG Practice Bulletin. Gestational Hypertension and Preeclampsia. ACOG Practice Bulletin [Internet]. 2020; 135:[e 237-60 pp.].

\section{Figures}




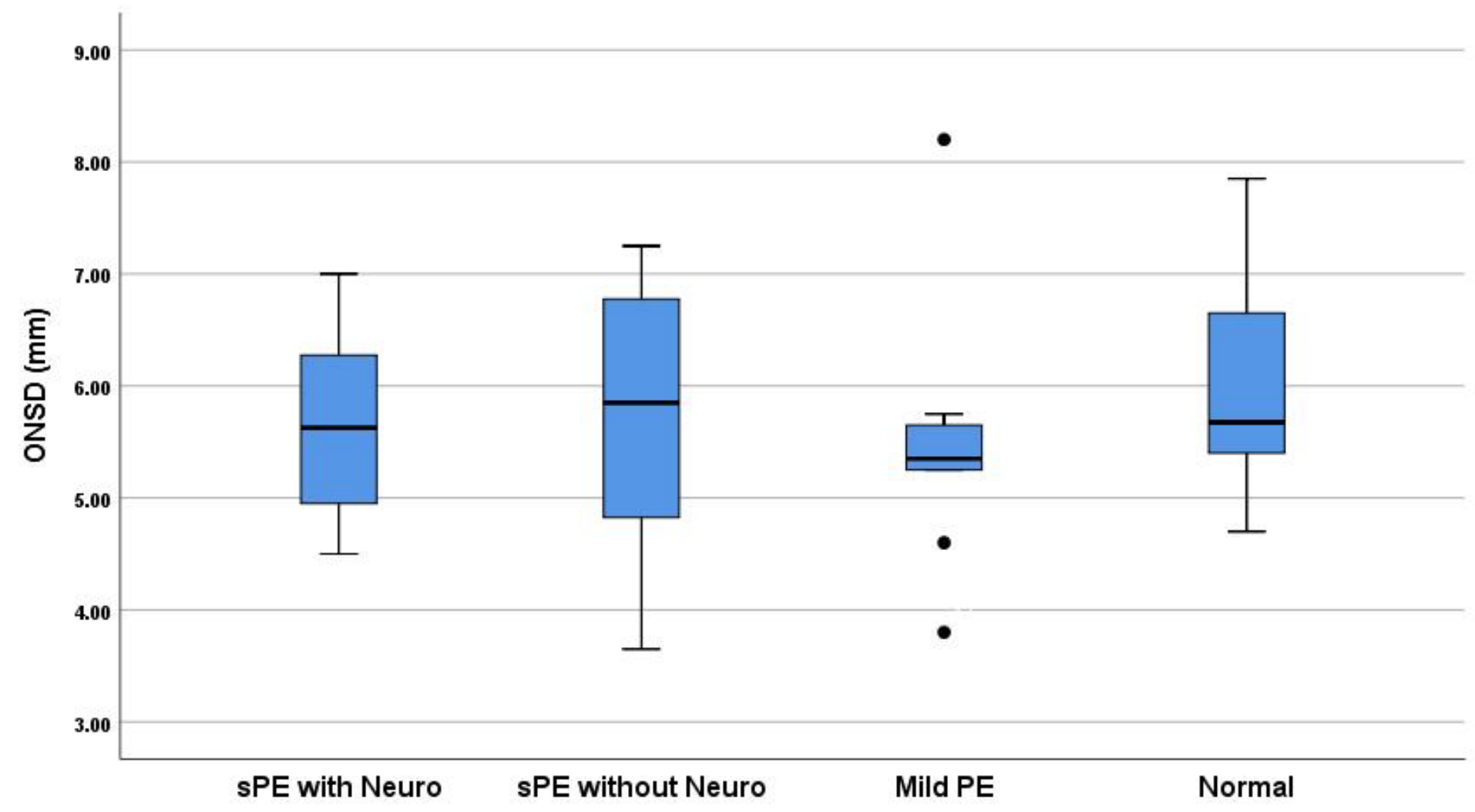

Figure 1

Distribution of Optic Nerve Sheath Diameters

ONSD: Optic nerve sheath diameters $(\mathrm{mm})$. sPE with Neuro: Severe preeclampsia patients with neurologic features. SPE without Neuro: Severe preeclampsia patients without neurologic featues. Mild PE: Preeclampsia patients without severe features. Normal: Control patients. 